\title{
ON NEUTRAL ABSORPTION AND SPECTRAL EVOLUTION IN X-RAY BINARIES
}

\author{
J. M. Miller ${ }^{1}$, E. M. CACKetT ${ }^{1,3}$, and R. C. Reis ${ }^{2}$ \\ ${ }^{1}$ Department of Astronomy, University of Michigan, 500 Church Street, Ann Arbor, MI 48109-1042, USA; jonmm@umich.edu \\ 2 Institute of Astronomy, University of Cambridge, Madingley Road, Cambridge CB3 OHA, UK \\ Received 2009 June 12; accepted 2009 October 13; published 2009 November 30
}

\begin{abstract}
Current X-ray observatories make it possible to follow the evolution of transient and variable X-ray binaries across a broad range in luminosity and source behavior. In such studies, it can be unclear whether evolution in the low-energy portion of the spectrum should be attributed to evolution in the source, or instead to evolution in neutral photoelectric absorption. Dispersive spectrometers make it possible to address this problem. We have analyzed a small but diverse set of X-ray binaries observed with the Chandra High Energy Transmission Grating Spectrometer across a range in luminosity and different spectral states. The column density in individual photoelectric absorption edges remains constant with luminosity, both within and across source spectral states. This finding suggests that absorption in the interstellar medium strongly dominates the neutral column density observed in spectra of X-ray binaries. Consequently, evolution in the lowenergy spectrum of X-ray binaries should properly be attributed to evolution in the source spectrum. We discuss our results in the context of X-ray binary spectroscopy with current and future X-ray missions.
\end{abstract}

Key words: black hole physics

\section{INTRODUCTION}

X-ray binaries show a remarkable variety of extreme phenomena, including rapid variability in many wavelength bands, the production of relativistic jets and hot winds, and outbursts that span factors of $10^{6}$ in luminosity (for a review of black hole transients, see Remillard \& McClintock 2006). Monitoring observations with the Rossi X-ray Timing Explorer and Swift enable the evolution of X-ray spectral and timing properties of $\mathrm{X}$-ray binaries to be traced in order to understand the accretion process (see, e.g., Rykoff et al. 2007). Model degeneracies that may occur in fitting a single spectrum can often be resolved by examining how multiple spectra evolve with time.

Major changes within the outbursts of transient and persistent $\mathrm{X}$-ray binaries are typically linked with changes in the nature of the accretion disk (see, e.g., Esin et al. 1997; Homan et al. 2001; also see Miller et al. 2006a). Tracking accretion disk evolution is therefore a primary goal of monitoring observations. The disk is directly visible in soft X-rays in black hole X-ray binaries, and likely also in neutron star binaries (e.g., Cackett et al. 2008a). However, the K-shell photoelectric absorption edges from most abundant elements fall below $2 \mathrm{keV}$, as do Fe L-shell edges. This absorption can complicate efforts to study disk properties, especially when using a spectrometer with limited energy range and resolution.

Prominent theoretical treatments of accretion flows predict modest winds (e.g., Begelman et al. 1983) and/or outflows with a high ionization parameter (e.g., Narayan \& Raymond 1999). Observationally, even the most extreme winds detected in X-ray binaries do not appear to generate enough column density to add appreciably to the neutral absorption column (Miller et al. 2006a, 2006b). Nevertheless, it is not uncommon to allow the equivalent neutral hydrogen column density to vary in modeling multiple spectra of an X-ray binary (see, e.g., Brocksopp et al. 2006; Caballero-Garcia et al. 2009; Cabanac et al. 2009). Even in cases where the neutral column density is held constant or jointly determined, this method is adopted somewhat arbitrarily, without specific reference to an observational or theoretical basis

\footnotetext{
3 Chandra Fellow.
}

(see, e.g., Sobczak et al. 2000; Miller et al. 2001; Nowak et al. 2002; Park et al. 2004; Gierlinski \& Done 2004; Kalemci et al. 2005; Rykoff et al. 2007).

Dispersive X-ray spectroscopy can separate variations in absorption from variations in the source spectrum. Even the resolution afforded by CCD spectrometers blends many weak edges into the continuum, and the optical depth and wavelength of stronger edges can be difficult to measure precisely. For instance, in fits to CCD spectra, the multiple Fe L edges can be modeled satisfactorily in terms of a single step function. In contrast, Chandra High Energy Transmission Grating Spectrometer observations of X-ray binaries have resolved the individual Fe L edges and their detailed properties (see, e.g., Schulz et al. 2002; Juett et al. 2006). Beyond simple edges, dispersive X-ray spectroscopy has clearly revealed the neutral O I $1 s-2 p$ resonance absorption line that was first glimpsed with the crystal spectrometer aboard Einstein (Schattenberg \& Canizares 1986; Paerels et al. 2001).

In this Letter, we extend recent work using high-resolution $\mathrm{X}$-ray spectroscopy by examining the evolution of neutral absorption in a sample of X-ray binaries. We find no evidence for variable absorption, consistent with an interstellar origin for neutral absorption.

\section{DATA SELECTION AND REDUCTION}

Our analysis is restricted to observations made with the Chandra High Energy Transmission Grating Spectrometer. The HETGS offers better resolution than the XMM-Newton Reflection Grating Spectrometer, as well as coverage across the full $0.3-10.0 \mathrm{keV}$ band. When the equivalent neutral hydrogen density is too high, much of the source continuum spectrum is scattered out of the line of sight. This must be avoided as absorption features are measured relative to a continuum. A number of clear absorption edges are detected in Cygnus X-1, which has an equivalent neutral hydrogen column density of $N_{H}=6.2 \times 10^{21} \mathrm{~cm}^{-2}$ (Schulz et al. 2002). However, an estimated column density of $8.0 \times 10^{21} \mathrm{~cm}^{-2}$ does not allow the absorption edges in XTE J1550-564 to be studied in detail (Miller et al. 2003). We therefore restricted 
Table 1

Source and Observation Parameters

\begin{tabular}{|c|c|c|c|c|c|c|c|}
\hline Source Name & $P_{\text {orb }}$ (days) & Distance $(\mathrm{kpc})$ & Companion & ID & Start (MJD) & Exposure (ks) & State \\
\hline GX 339-4 & $1.76^{\mathrm{a}}$ & $8.5^{\mathrm{b}}$ & Low mass ${ }^{\mathrm{a}}$ & $\begin{array}{l}4420 \\
4569 \\
4570\end{array}$ & $\begin{array}{l}52715.8 \\
53239.5 \\
53282 .\end{array}$ & $\begin{array}{l}76.2 \\
50.1 \\
50.2\end{array}$ & $\begin{array}{c}\text { Hard/intm. } \\
\text { High/soft } \\
\text { High/soft }\end{array}$ \\
\hline XTE J1817-330 & short $^{\mathrm{c}}$ & $8.5^{\mathrm{d}}$ & Low mass ${ }^{\mathrm{c}}$ & $\begin{array}{l}6615 \\
6616 \\
6617 \\
6618 \\
\end{array}$ & $\begin{array}{l}53779.1 \\
53790.2 \\
53809.3 \\
53877.6\end{array}$ & $\begin{array}{l}50.0 \\
49.6 \\
46.7 \\
51.0\end{array}$ & $\begin{array}{l}\text { High/soft }{ }^{1} \\
\text { High/soft }^{1} \\
\text { High/soft } \\
\text { High/soft }\end{array}$ \\
\hline Cygnus $\mathrm{X}-1$ & $5.6^{\mathrm{e}}$ & $2.5^{\mathrm{f}}$ & $09.7 \mathrm{Iab}^{\mathrm{e}}$ & $\begin{array}{l}3724 \\
3815 \\
\end{array}$ & $\begin{array}{l}52485.7 \\
52702.7 \\
\end{array}$ & $\begin{array}{l}26.4 \\
55.9 \\
\end{array}$ & $\begin{array}{l}\text { High/soft }{ }^{\mathrm{m}} \\
\text { Low/hard }\end{array}$ \\
\hline $4 \mathrm{U} 1820-30$ & $0.007^{\mathrm{g}}$ & $7.6^{\mathrm{h}}$ & Low mass ${ }^{g}$ & $\begin{array}{l}6633 \\
6634 \\
7032 \\
\end{array}$ & $\begin{array}{l}53959.9 \\
54028.2 \\
54044.6\end{array}$ & $\begin{array}{l}25.2 \\
25.1 \\
46.3 \\
\end{array}$ & $\begin{array}{l}\mathrm{BB}^{\mathrm{p}} \\
\mathrm{BB}^{\mathrm{p}} \\
\mathrm{BB}^{\mathrm{l}}\end{array}$ \\
\hline Cygnus $\mathrm{X}-2$ & $9.843^{\mathrm{i}}$ & $7.2^{\mathrm{j}}$ & $\mathrm{A} 5-\mathrm{F} 2^{\mathrm{i}}$ & $\begin{array}{l}8170 \\
8599\end{array}$ & $\begin{array}{l}54337.7 \\
54335.2\end{array}$ & $\begin{array}{l}78.7 \\
70.6\end{array}$ & $\begin{array}{l}\mathrm{NB}, \mathrm{HB}, \mathrm{FB}^{\mathrm{q}} \\
\mathrm{NB}, \mathrm{HB}, \mathrm{FB}^{\mathrm{q}}\end{array}$ \\
\hline
\end{tabular}

Notes. This table lists the name, orbital period, and the companion type (where known) for the X-ray binaries included in our sample. The Chandra observation identification number, observation start time, and exposure time are also given for each observation. Finally, the "state" in which the source was observed is provided. The first three sources are black hole candidates, the last two sources are low-mass X-ray binaries harboring neutron stars.

${ }^{\text {a }}$ Hynes et al. (2003).

${ }^{b}$ Zdziarski et al. (2004).

c Torres et al. (2006).

${ }^{\mathrm{d}}$ Based on its coordinated near to the Galactic center.

${ }^{\text {e }}$ Gies \& Bolton (1982).

${ }^{\mathrm{f}}$ Bregman et al. (1983).

${ }^{\mathrm{g}}$ Stella et al. (1987).

${ }^{\text {h }}$ Kuulkers et al. (2003).

${ }^{\mathrm{i}}$ Cowley et al. (1979).

${ }^{j}$ Kuulkers et al. (1999).

${ }^{\mathrm{k}}$ Miller et al. (2004).

${ }^{1}$ This work.

${ }^{\mathrm{m}}$ Feng et al. (2003).

${ }^{n}$ Miller et al. (2006a).

p "Banana" branch; Cackett et al. (2009).

${ }^{\mathrm{q}}$ Normal branch, horizontal branch, flaring branch; Schulz et al. (2009).

our analysis to sources equal to or below approximately $6 \times 10^{21} \mathrm{~cm}^{-2}$.

Our analysis was also restricted by the need to select variable and transient sources. Observations of transient sources with large missions can be difficult, and relatively few sources with low or moderate column densities have been observed on multiple occasions. The sources and observations selected for our analysis are listed in Table 1. Different source types, companion types, and orbital periods are included in the sample.

When observing bright sources using the HETGS, the ACIS$\mathrm{S}$ array of CCDs must be run in "continuous clocking" mode to prevent photon pileup. For consistency, the observations considered in this work employed this observing mode. The only exception is a single observation of GX 339-4 in a low flux state (obsid 4420; see Table 1). An observation of Cygnus X-1 in the low/hard state that was not taken in the "continuous clocking" mode (obsid 107) suffered heavy photon pileup, complicating absorption spectroscopy (see Juett et al. 2004), and was therefore rejected. For more information on the specific nature of HETGS observations made in the "continuous clocking" mode, please see Miller et al. (2006b) or Cackett et al. (2008b).

Most of the spectra considered in this work were obtained using the "TGCat" data center (see http://tgcat.mit.edu). This facility is run by the Chandra X-ray Center and provides firstorder HETGS spectra and responses derived using up-to-date CIAO software and calibrations. An error in the instrument offsets used to observe XTE J1817-330 required custom processing as per the procedure outlined in Miller et al. (2006b) and Cackett et al. (2008b). Two observations of 4U 1820-30 were previously reduced and analyzed by Cackett et al. (2008b) (obsid 6633 and 6634), and the same spectra are used in this work. Using CIAO version 4.0.2, we combined the first-order MEG spectra and instrument response files using the CIAO tool "add_gratings_spectra." The same procedure was repeated for the first-order HEG spectra. Again owing to the pointing error, only the minus-side first-order spectra of XTE J1817-330 were used in our analysis. To create distinct spectra of Cygnus X-2 in the flaring, horizontal, and normal branches of its " $\mathrm{Z}$ " track, we employed exactly the same selection criteria and procedure as Schulz et al. (2009) to both observations (obtaining a total of six spectra). The "aglc" script was used in the ISIS analysis package (Houck \& Denicola 2000) to create light curves and color-color diagrams that were used to create spectra from each branch.

All of the spectral fits reported in this work were made using XSPEC version 12 (Arnaud \& Dorman 2000). Unless otherwise stated, errors are $1 \sigma$ errors.

\section{ANALYSIS AND RESULTS}

The true neutral absorption column density observed in a given spectrum should not be a function of the continuum flux 
model. However, to ensure model-independent results, we fit for prominent neutral photoelectric absorption edges individuallyin narrow wavelength ranges-using local power-law models for the continuum. The $\mathrm{O} \mathrm{K}, \mathrm{Fe} \mathrm{L}$, and $\mathrm{Ne} \mathrm{K}$ edges were fit in the 20-25 $\AA, 16-18 \AA$, and 13-15 $\AA$ bands, respectively. At longer wavelengths, the MEG has a higher effective area than the HEG; moreover, it covers longer wavelengths than the HEG. Fits to absorption edges were therefore made to the combined first-order MEG spectrum from each observation.

In many of the spectra, it was quickly apparent that simple step-function edge models do not fully describe the data. We therefore used the "tbnew" model (J. Wilms et al. 2009, in preparation), which includes up-to-date cross sections and detailed edge structure. A new functionality in "tbnew" can be used to fit the column density in an individual edge by setting the overall column density and abundance of all elements (apart from the element of interest) to zero, and restricting the column density to negative values for the parameter of interest. (Negative values instruct the model to return the column density for the element; positive values return the abundance of a given element relative to hydrogen.) All fitting parameters relating to grain properties were fixed at their default value. In our fits, then, "tbnew" functioned as a model with only one variable parameter (the column density of the element of interest). Due to a combination of a relatively high total absorption column and the particular choice of instrumental pointing offsets used, useful constraints on the $\mathrm{O} \mathrm{K}$ edge could not be obtained from existing data on GX 339-4, 4U 1820-30, and Cygnus X-2. We note that Yao et al. (2009) were able to study the Oxygen edge in Cygnus X-2 by adding multiple spectra, but this procedure is inconsistent with the aim of this study.

To estimate the luminosity of each source in a given observation, we fit the first-order HEG spectra in the $1.2-10.0 \mathrm{keV}$ range using simple absorbed continuum models. The equivalent neutral hydrogen column density was allowed to vary but values were found to be broadly consistent with fits to individual edges. These models should be regarded as fiducial. Some of the fits obtained are not formally acceptable, owing to calibration uncertainties and/or unrelated ionized absorption lines in the spectra. The black hole sources were fit with a continuum consisting of a disk blackbody and a power law. The neutron star spectra were all fit with a continuum consisting of a simple blackbody function and a power law. To represent the evolution of absorption with luminosity, the luminosity in each observation was calculated for a given distance. Errors on the luminosity were then calculated using the $1 \sigma$ errors on the continuum flux. The distances assumed in order to calculate luminosity values are listed in Table 1.

Some of the spectra modeled in this Letter have not been analyzed previously. All four Chandra observations of XTE J1817-330 were made in the high/soft state. This is evident from inner disk color temperatures that range between $k T=$ $0.48 \mathrm{keV}$ and $0.94 \mathrm{keV}$, and power law indices that range between $\Gamma=2.4$ and 4.0. The evolution of the bright phase of the outburst of XTE J1817-330 is detailed in Rykoff et al. (2007), and comparisons to that work again indicates that the Chandra observations were all made in high/soft states. Similarly, spectral results have not been reported based on fits to the second and third observations of GX 339-4 that are considered in this work (obsids 4569 and 4570). Here again, the flux was strongly dominated by the accretion disk, with inner disk color temperatures of $k T=0.68 \mathrm{keV}$ and $0.81 \mathrm{keV}$. Finally, the third observation of 4 U 1820-30 (obsid 7032) has not been

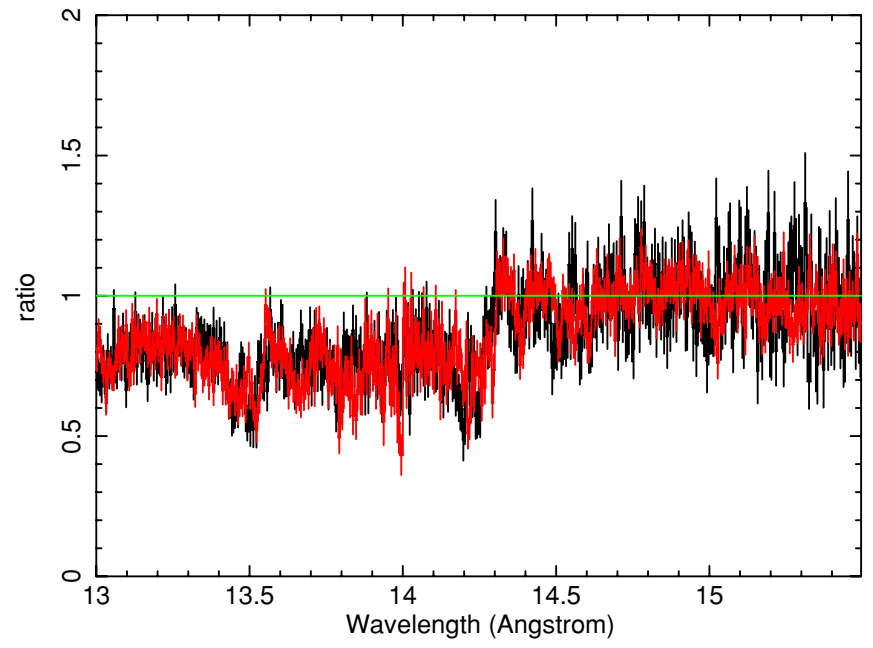

Figure 1. In this plot, high/soft (black) and low/hard (red) spectra of Cygnus $\mathrm{X}-1$ in the region of the $\mathrm{Ne} \mathrm{K}$ edge are plotted as a ratio to a power-law continuum. The best-fit column density for the edge has been set to zero to show the remarkable consistency of the edge in different spectral states.

published previously, but analysis as per Cackett et al. (2008b, 2009) suggests that the source was observed in the "banana branch."

We find that neutral absorption is remarkably constant with source luminosity, and consistent across different spectral states. Figure 1 shows the Ne Kedge in the high/soft and low/hard state spectra of Cygnus X-1. The jump in the continuum at the Ne K edge is consistent. Figure 2 plots the column density measured in individual absorption edges versus luminosity for the sources in our sample. For the black hole sources, the column density in a given edge is constant with luminosity within $1 \sigma$ errors. The case is much the same with the neutron star X-ray binaries. The column density measured in the Ne K edge of $4 \mathrm{U} 1820-30$ is marginally different at the $1 \sigma$ level in two observations, but both are consistent with a third observation. All three columns agree at the $90 \%$ confidence level. The same holds true for two observations of Cygnus X-2.

The Ne column densities that we have measured agree with values reported by Juett et al. (2004) for 4U 1820-30, Cygnus $\mathrm{X}-2$, and GX 339-4. The Ne column density that we measured in Cygnus X-2 also agrees with a detailed analysis made by Yao et al. (2009). Our value of the Ne column density agrees with that measured in Cygnus X-1 by Hanke et al. (2009) though our values of the $\mathrm{O}$ and $\mathrm{Fe}$ columns differ by factor of approximately 2 . These disparities can likely the result of different continuum modeling procedures and implementations of "tbnew."

\section{DISCUSSION AND CONCLUSIONS}

To better understand the evolution of the low-energy spectrum of X-ray binaries, we made fits to individual photoelectric absorption edges in high-resolution X-ray spectra of selected sources. The column density measured in individual edges is not observed to vary across different spectral states, nor over a broad range in luminosity (see Table 1 and Figure 2). This suggests that gas from X-ray binaries is not typically an important source of the neutral absorption observed in the spectra of these systems. Rather, neutral absorption must be dominated by the interstellar medium (ISM). A similar conclusion was reached by Juett et al. (2004) based on upper limits on the velocity dispersion of the ISM as measured through absorption lines. Evolution in the low- 


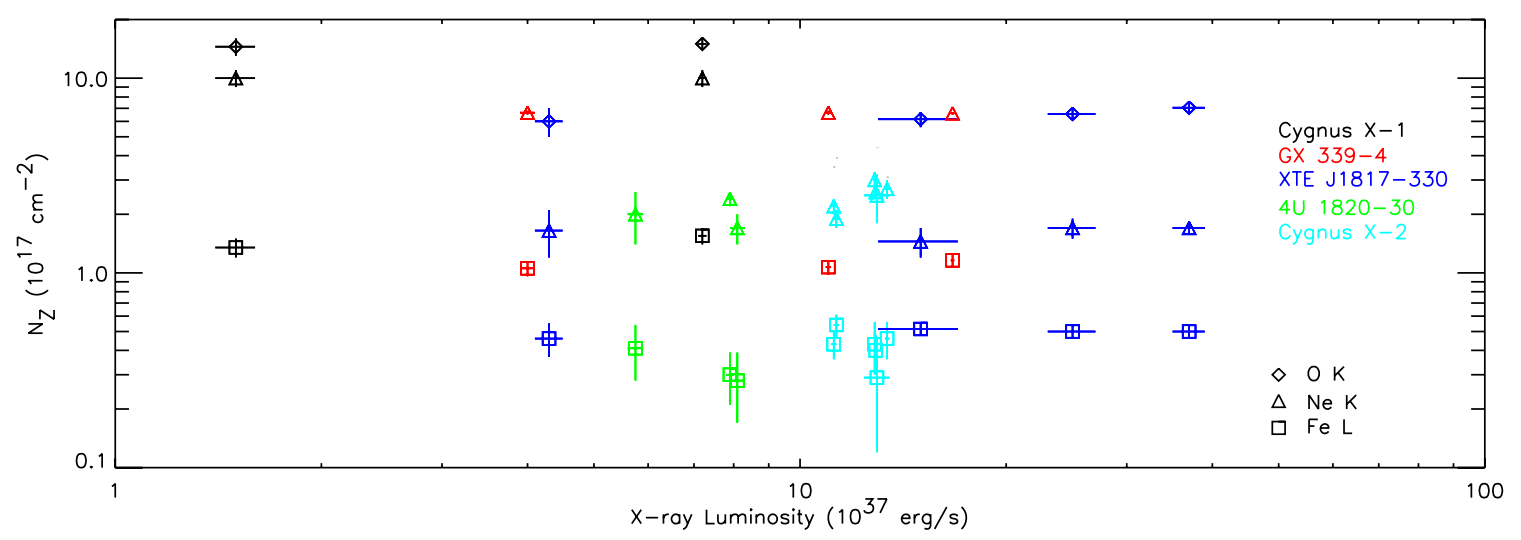

Figure 2. Plot shows the column density measured in specific low-energy absorption edges vs. luminosity, for black hole and neutron star X-ray binaries. Errors on column density are $1 \sigma$ errors.

energy spectrum of typical X-ray binaries, then, is best attributed to evolution in the source continuum.

Neutral absorption in X-ray spectra is often fit by a single model that parameterizes the accumulated absorption from individual edges as an equivalent neutral hydrogen column density. Values obtained from high-resolution spectra are likely to give the best measure of a true equivalent total column density. In practice, instrumental problems such as internal scattering, carbon buildup from optical blocking filters, and gain drift could prevent the adoption of a gratings-derived value for the neutral column. In such cases, our results suggest that a single value of the equivalent neutral hydrogen column density should be used to fit multiple spectra from monitoring observations of a given source with a given detector.

The outflows that are observed in X-ray binaries are highly ionized-dominated by He-like and H-like charge states (see, e.g., Lee et al. 2002; Miller et al. 2004, 2006b; Schulz et al. 2008). An especially dense wind was observed in GRO J1655-40, and even in that case the ionized columns observed are insufficient to create strong absorption edges that could be mistaken for additional neutral absorption (Miller et al. 2006c, 2008). Moreover, in sources such as H 1743-322, GRO J1655-40, and GRS 1915+105, a paradigm is emerging wherein ionized winds are active in soft, disk-dominated states, but absent in spectrally hard states like those that typically hold when sources accrete at a low fraction of their Eddington limit (Miller et al. 2006b, 2006c, 2008; Neilsen \& Lee 2009). In Figure 2, it is clear that any variation in ionized winds across states does not affect the column density measured in neutral edges.

Our results are based on spectra which only reach down to approximately $0.01 L_{\text {Edd }}$. Sensitive high-resolution X-ray spectra that would permit strong constraints on absorption variability have not yet been obtained from sources at lower luminosity. However, theoretical arguments again point to ionized outflows that would contribute little to a neutral column. Winds from advection-dominated accretion flows are expected to be extremely hot (since advective flows are very hot), and line spectra should be dominated by He-like and H-like charge states (e.g., Narayan \& Raymond 1999). Recent observations of the stellarmass black hole V404 Cyg, which accretes at about $10^{-5} L_{\text {Edd }}$, are able to rule out the winds predicted by some advective models (Bradley et al. 2007). This further suggests that ionized outflows are not likely to contribute significantly to line-of-sight absorption, even as sources approach quiescence.

There are at least two classes of sources where our results may not hold in all circumstances. Neutron star X-ray binaries known as "dippers" are viewed at high inclinations, and material in the outer disk can block emission from the central engine (see, e.g., Diaz Trigo et al. 2006). Within such flux dips, the observed neutral absorption column may vary due to the changing geometry within the binary. Similarly, winds from massive stars are known to be clumpy and to sometimes cause flux dips; some of these dips may also cause variations in the observed equivalent neutral hydrogen column density (e.g., Balucinska-Church et al. 2000).

We thank Joern Wilms for creating the "tbnew" model used in this work, and for guidance on its implementation. We acknowledge helpful comments from the anonymous referee that improved this Letter. J.M.M. gratefully acknowledges funding from the Chandra Guest Observer program. E.M.C. gratefully acknowledges support provided by NASA through the Chandra Fellowship Program, grant number PF8-90052. R.C.R. acknowledges STFC for financial support.

\section{REFERENCES}

Arnaud, K. A., \& Dorman, B. 2000, XSPEC is available via the HEASARC online service, provided by NASA/GSFC

Balucinska-Church, M., et al. 2000, MNRAS, 311, 861

Begelman, M. C., McKee, C. F., \& Shields, G. A. 1983, ApJ, 271, 70

Bradley, C. K., et al. 2007, ApJ, 667, 427

Bregman, J., et al. 1983, ApJ, 185, L117

Brocksopp, C., et al. 2006, MNRAS, 365, 1203

Caballero-Garcia, M. D., et al. 2009, ApJ, 692, 1339

Cabanac, C., Fender, R. P., Dunn, R. J. H., \& Kording, E. G. 2009, MNRAS, 396, 1415

Cackett, E. M., et al. 2008a, ApJ, 674, 415

Cackett, E. M., et al. 2008b, ApJ, 677, 1233

Cackett, E. M., et al. 2009, ApJ, 690, 1847

Cowley, A. P., Crampton, D., \& Hutchings, J. B. 1979, ApJ, 231, 539

Diaz Trigo, M., et al. 2006, A\&A, 445, 179

Esin, A. A., McClintock, J. E., \& Narayan, R. 1997, ApJ, 489, 865

Feng, Y. X., Tennant, A. F., \& Zhang, S. N. 2003, ApJ, 597, 1017

Gierlinski, M., \& Done, C. 2004, MNRAS, 347, 885

Gies, D. R., \& Bolton, C. T. 1982, ApJ, 260, 240

Hanke, M., Wilms, J., Nowak, M. A., Pottschmidt, K., Schulz, N. S., \& Lee, J. C. 2009, ApJ, 690, 330

Homan, J., et al. 2001, ApJS, 132, 377

Houck, J. C., \& Denicola, L. A. 2000, in ASP Conf. Ser. 216, Astronomical Data Analysis Software and Systems IX, ed. N. Manset, C. Veillet, \& D. Crabtree (San Francisco, CA: ASP), 591

Hynes, R. I., Steeghs, D., Casares, J., Charles, P., \& O’Brien, K. 2003, ApJ, 583, L95

Juett, A. M., Schulz, N. S., \& Chakrabarty, D. 2004, ApJ, 612, 308

Juett, A. M., Schulz, N. S., Chakrabarty, D., \& Gorczyca, T. W. 2006, ApJ, 648, 1066

Kalemci, E., et al. 2005, ApJ, 622, 508

Kuulkers, E., Wijnands, R., \& van der Klis, M. 1999, MNRAS, 308, 485 
Kuulkers, E., et al. 2003, A\&A, 399, 663

Lee, J. C., et al. 2002, ApJ, 567, 1102

Miller, J. M., et al. 2001, ApJ, 546, 1055

Miller, J. M., et al. 2003, MNRAS, 338, 7

Miller, J. M., et al. 2004, ApJ, 601, 450

Miller, J. M., et al. 2006a, ApJ, 653, 525

Miller, J. M., et al. 2006b, ApJ, 646, 394

Miller, J. M., et al. 2006c, Nature, 441, 953

Miller, J. M., et al. 2008, ApJ, 680, 1359

Narayan, R., \& Raymond, J. 1999, ApJ, 515, L69

Neilsen, J., \& Lee, J. C. 2009, Nature, 458, 481

Nowak, M. A., Wilms, J., \& Dove, J. B. 2002, MNRAS, 332, 856

Paerels, F., et al. 2001, ApJ, 546, 338

Park, S. Q., et al. 2004, ApJ, 610, 378
Remillard, R. A., \& McClintock, J. E. 2006, ARA\&A, 44, 49

Rykoff, E. S., Miller, J. M., Steeghs, D., \& Torres, M. A. P. 2007, ApJ, 666, 1129

Schattenberg, M. L., \& Canizares, C. R. 1986, ApJ, 301, 759

Schulz, N. S., Cui, W., Canizares, C. R., Marshall, H. L., Lee, J. C., Miller, J. M., \& Lewin, W. H. G. 2002, ApJ, 565, 1141

Schulz, N. S., Kallman, T. E., Galloway, D. K., \& Brandt, W. N. 2008, ApJ, 672,1091

Schulz, N. S., et al. 2009, ApJ, 692, L80

Sobczak, G. J., et al. 2000, ApJ, 544, 993

Stella, L., Priedhorsky, W., \& White, N. E. 1987, ApJ, 312, L17

Torres, M., et al. 2006, ATEL, 733

Yao, Y., et al. 2009, ApJ, 692, L80

Zdziarski, A. A., et al. 2004, MNRAS, 351, 791 